\title{
Obtención de inferencias en documentos de pasantía descritos a nivel ontológico
}

Obtaining inferences in documents internship described ontological level

\author{
Obtenção de inferências em documentos de estágio descritos a nível ontológico
}

Germán Alejandro Pinilla-García ${ }^{1}$, Julio Barón-Velandia ${ }^{2}$

Forma de citar: G. A. Pinilla-García, J. Barón-Velandia, "Obtención de inferencias en documentos de pasantía descritos a nivel ontológico", Respuestas, vol. 20, no. 2, pp. 119-134, 2015.

Recibido:

Diciembre 1 de 2014

Aceptado:

Junio 4 de 2015
${ }^{1}$ Ingeniería de Sistemas (c) gapinillag@correo.udistrital.edu.co ORCID: 0000-0002-8924-3396 Universidad Distrital Francisco José de Caldas Bogotá - Colombia

${ }^{2}$ Maestría en Teleinformática jbaron@udistrital.edu.co ORCID: 0000-0002-9491-5564 Universidad Distrital Francisco José de Caldas Bogotá - Colombia

\section{Resumen}

Antecedentes: El número de usuarios que en la actualidad se apropia de prácticas de consulta utilizando sistemas documentales crece de manera significativa, sin embargo el uso convencional de procesos de búsqueda de manera textual o sintáctica, produce una extensa cantidad de resultados con bajo nivel de pertinencia, obligando al usuario a emplear un gran número de horas para acceder a los resultados proporcionados y determinar aquellos que presentan un alto grado de correspondencia con su área de interés. Objetivo: El objetivo fundamental de este trabajo es obtener un conjunto de inferencias mediante la lógica de predicados, a partir de un modelo ontológico de trabajos de grado en modalidad pasantía, las cuales permitan obtener información implícita a partir de las descripciones semánticas que representan a cada uno de los documentos referenciados. Metodología: El modelo ontológico se obtuvo consultando las normas y disposiciones acerca de pasantías, estableciendo los conceptos del dominio y las correspondientes relaciones entre conceptos; la representación computacional del modelo se realizó en lenguaje OWL y mediante lógica de predicados de primer orden, se establecieron las sentencias que permiten la realización de inferencias para obtener información implícita contenida en la base de conocimiento. Resultados: Entre las inferencias más significativas que se obtuvieron están: la tipificación de documentos relacionados con un tema de interés y la identificación de docentes por áreas de experticia. Conclusión: La aplicación de la lógica de predicados a esta modalidad de trabajos de grado permite realizar inferencias de manera dinámica sobre el contenido representado a nivel ontológico y puede utilizarse para complementar la información descrita explícitamente en el modelo, con respecto a temáticas y áreas de experticia de los docentes, independientemente de la ubicación de los repositorios de documentos. Las sentencias utilizadas pueden ser aplicadas a otras modalidades de grado como investigación y monografía.

Palabras clave: Inferencia, lógica de predicados, ontología, pasantía.

\begin{abstract}
Background: The number of users that currently appropriates query practices using document systems has grown significantly, however, the conventional use of textual or syntactical search processes produces a vast amount of low-relevant results, forcing the user to employ a large number of hours to access the results provided and to determine those with a high level of correspondence with their field of interest. Objective: The main
\end{abstract}


objective of this work was to obtain a set of inferences through predicate logic, from an internship documents ontological model, which allow to obtain implicit information from semantic descriptions representing each one of the referenced documents. Methods: The ontological model was obtained by consulting standards and provisions about internships, setting the domain concepts and the corresponding relations between concepts; the model computational representation was developed with OWL language and first-order predicate logic were used to establish the sentences that allow making inferences, to get implicit information in the knowledge base. Results: Among the most significant inferences that were obtained are: the classification of documents related to a topic of interest and the identification of teachers by areas of expertise. Conclusion: The application of predicate logic to internship documents allows to dynamically make inferences about the content represented at ontological level and can be used to complement the explicitly described information in the model, regarding issues and areas of expertise of the teachers, regardless of the location of document repositories. The statements used can be applied to other types of degree as research and monography.

Keywords: Inference, predicate logic, ontology, internship.

\section{Resumo}

Antecedentes: $\mathrm{O}$ número de usuários que na atualidade se apropria de práticas de consulta utilizando sistemas de documentação cresce de maneira significativa. No entanto, o uso convencional de processos de busca de maneira textual o sintática, produz una extensa quantidade de resultados com baixo nível de pertinência, obrigando ao usuário a empregar um grande número de horas para aceder aos resultados proporcionados e determinar aqueles que apresentam um elevado grau de correspondência com sua área de interesse. Objetivo: O objetivo fundamental deste trabalho foi obter um conjunto de inferências mediante a lógica de predicados, a partir de um modelo ontológico de trabalhos de graduação em modalidade estágio, as quais permitam obter informação implícita a partir das descrições semânticas que representam a cada um dos documentos referenciados. Metodologia: O modelo ontológico se obteve consultando as regras e regulamentos sobre estágios, estabelecendo os conceitos do domínio e as correspondentes relações entre conceitos; a representação computacional do modelo se realizou numa linguagem OWL e mediante lógica de predicados de primeira ordem, se estabeleceram as sentencias que permitem a realização de inferências para obter informação implícita contida na base de conhecimento. Resultados: Entre as inferências mais significativas que se obtiveram estão: a tipificação de documentos relacionados com um tema de interesse e a identificação de docentes por áreas de perícia. Conclusão: A aplicação da lógica de predicados nesta modalidade de trabalhos de graduação permite realizar inferências de maneira dinâmica sobre o conteúdo representado a nível ontológico e pode utilizar-se para complementar a informação descrita explicitamente no modelo, com respeito a temáticas e áreas de perícia dos docentes, independentemente da localização dos repositórios de documentos. As sentencias utilizadas podem ser aplicadas a outras modalidades de graduação como pesquisa e monografia.

Palavras-chave: Estágio, inferência, lógica de predicados, ontologia.

\section{Introducción}

El proceso de búsqueda de documentos de grado en modalidad pasantía en sistemas documentales se lleva a cabo mediante palabras clave, utilizando técnicas de consulta textual o sintáctica que generan un alto número de resultados, desde donde el usuario debe seleccionar aquellos que correspondan con sus áreas de interés, entre las propuestas 
destacadas para el manejo de este tipo de documentos están: la base de datos de Tesis Españolas Ordenadas (Teseo, por sus siglas en español)[1], implementada por el Ministerio de Educación en España, mediante el registro de campos de datos sistémicos, disponibles en Tesauros o vocabularios controlados. La búsqueda realizada en esta herramienta proporciona resultados según la(s) palabra(s) ingresada(s) de manera textual mediante campos como: autor, director o universidad de procedencia, omitiendo varios documentos que puedan estar relacionados aunque no estén referenciados con las palabras incluidas. A nivel latinoamericano, entre las iniciativas destacadas está Cybertesis [2], cuyo objetivo es la difusión de tesis electrónicas de instituciones de Europa, África y América, donde las consultas pueden ser realizadas mediante el ingreso de datos como: autor, tema o título. En Colombia se destacan iniciativas como la promovida por la Universidad Nacional, que cuenta con una base de datos conocida como Sistema Nacional de Bibliotecas (SINAB, por sus siglas en español) [3], la cual permite consultar documentos como revistas o trabajos de grado propuestos en todas las sedes a nivel nacional. La iniciativa propuesta desde el año 2009 por la Universidad Industrial de Santander busca la sistematización de trabajos de grado, permitiendo la consulta mediante el ingreso de datos principales como el tema o el autor. Las propuestas de sistemas documentales descritas anteriormente almacenan e indexan información bajo el esquema de motores de bases de datos relacionales, con lo cual la búsqueda se realiza con base en la sintaxis del contenido de cada registro existente. A nivel de representación semántica se encontró una propuesta que describe trabajos de grado mediante lenguaje ontológico OWL y su respectiva consulta en lenguaje SPARQL [4]. Esta nueva forma de modelar sistemas documentales mejora su representación, desaprovechando la posibilidad de realizar deducciones con base en las relaciones existentes entre los conceptos, de manera que se pueda incluir en los resultados conceptos asociados a los conceptos principales, los cuales son descritos mediante palabras clave. ¿Cómo mejorar la pertinencia de los resultados obtenidos por el usuario al consultar repositorios de documentos de pasantía, que cuentan con descripción a nivel ontológico? El objetivo fundamental de este trabajo es obtener un conjunto de inferencias mediante la lógica de predicados, a partir de un modelo ontológico de trabajos de grado en modalidad pasantía, que permitan obtener información implícita a partir de las descripciones semánticas que representan a cada uno de los documentos referenciados. La gestión de este tipo de documentos representa para las instituciones una ventaja ya que pueden generar nuevos proyectos con base en los anteriores, al contar con un modelo de inferencias se amplían las posibilidades de relacionar áreas de trabajo con proyectos y expertos más allá de las descripciones sintácticas con que se cuenta en sistemas convencionales. La estructura de este documento se conforma de los siguientes apartados: La sección 2.2 presenta la metodología utilizada y las fases generales. La sección 2.2.1 presenta la descripción de los conceptos importantes de la ontología. En la sección 2.2.2 se hace uso del lenguaje OWL para representar la ontología presentada en la sección 2.2.1. En la sección 2.3 se utiliza la lógica de predicados de primer orden para representar las reglas correspondientes a la ontología. Finalmente, la sección 3 presenta las inferencias obtenidas a partir de las reglas.

\section{Materiales y métodos}

\subsection{Fundamentos Teóricos}

\subsubsection{Ontología}

Las ontologías son una herramienta flexible en la representación del conocimiento, definidas como "un conjunto de términos de conocimiento, que incluye un vocabulario, relaciones y un conjunto de reglas lógicas y de inferencia sobre un domino en particular" [5]. Desde las ciencias de la computación, se define como "una especificación explícita de
Julio - Diciembre 2015 ISSN 0122-820X E-ISSN 2422-5053 PP: 119-134 
No. 2

Julio - Diciembre 2015 ISSN 0122-820X E-ISSN 2422-5053 PP: 119-134 una conceptualización" [6]. Una ontología se compone de elementos como las clases o conceptos del dominio, propiedades que se representan mediante relaciones o atributos, también consta de individuos o instancias de las mismas clases y axiomas los cuales son restricciones determinadas para uno o más elementos de la ontología. Para conformarla es importante el lenguaje de representación formalizado, reflejado en la organización de las clases en forma jerárquica, con el objetivo de aplicar la relación clase-subclase, estableciendo una aproximación multimodal con propiedades de su superclase. Es así como se identifica una ontología específica a un grupo de individuos [7]. Finalmente, se señala a la ontología, como una especificación compartida de un dominio de conocimiento que permite la comunicación entre personas y sistemas heterogéneos [8]. Por otro lado los componentes de la atmósfera de la ontología han mostrado la representación computacional en el contexto OWL, el cual es un lenguaje desarrollado por el W3C [9] Web Ontology Working Group, el cual se creó como una extensión de Resource Description Framework (RDF, por sus siglas en inglés) y se utiliza en el desarrollo de ontologías y el uso compartido por la web (web semántica) [10], permitiendo especificar las características como instancias y sus interrelaciones.

Para este trabajo se hizo uso de un modelo propuesto acerca de la pasantía como modalidad de grado, el cual está compuesto de entidades como Estudiante y Director, las cuales representan conceptos, estos a su vez están interrelacionados mediante términos dentro de los que están evaluadoPor y validadoPor, entre otros. Este modelo es descrito mediante OWL, lo cual permite especificar las reglas que describen las restricciones entre conceptos y de esta manera obtener uno que sea correcto y ajustado a las características de la pasantía, realizando inferencias que contengan información implícita en el mismo.

\subsubsection{Lógicas descriptivas}

Son un conjunto de representaciones formales, las cuales surgieron como un modelo de representación terminológica de un dominio de interés de manera taxonómica [11]. Las lógicas descriptivas pueden ser aplicadas a nivel abstracto evaluando las relaciones conceptuales y a nivel concreto procesando las instancias disponibles, dando lugar a:

\section{T-box:}

Para la descripción de conceptos jerárquicos o propiedades generales de los conceptos

\section{A-box:}

Para establecer la relación de cada una de las instancias de los conceptos con su ubicación en la jerarquía [12].

Cada una de las afirmaciones está representada por predicados que tienen un valor de verdadero o falso [13]. Esta lógica tiene un alto grado de expresividad, permitiendo así representar el dominio de los trabajos de grado de una universidad. Entiéndase la información como una colección de metadatos categorizada como información semántica o instructiva [14]. A nivel semántico, estas lógicas abarcan la lógica de predicados de primer orden, la cual permite representar información más detalladamente y a la vez obtener inferencias más precisas basadas en un modelo descrito mediante lenguajes como OWL.

\subsubsection{Lógica de predicados de primer orden}

En particular, la lógica de predicados de orden cero está limitada a afirmaciones o proposiciones que tienen un valor de verdad definido, sin embargo carece de estructuras como cuantificadores o variables que representen entidades, siendo imposible representar afirmaciones como "Todos los hombres son mortales" o "Aristóteles es un hombre". De esta forma se optó para este trabajo el uso de la lógica de predicados de primer orden, la cual permite investigar la estructura interna de las variables de tipo 
proposicional y tiene una semántica definida acorde con lo requerido. Adicionalmente, dentro de la inteligencia artificial esta lógica relacionada con las lógicas descriptivas se utiliza principalmente en el área de la representación del conocimiento [15].

La lógica de predicados de primer orden es un lenguaje formal que hace uso de elementos como constantes, variables, funciones $\mathrm{y}$ cuantificadores para representar hechos, los cuales forman el alfabeto de este lenguaje [16]. Los cuantificadores son símbolos matemáticos que especifican condiciones cuantitativas de un determinado conjunto indicando cuantos elementos poseen una propiedad. Esta lógica permite realizar deducciones mediante razonamientos de tipo $\left\{\mathrm{P}_{1}, \mathrm{P}_{2}, \mathrm{P}_{3} \ldots\right\} \rightarrow \mathrm{q}$, teniendo validez cuando la conclusión obtenida es una consecuencia de tipo lógico obtenida de las premisas evaluadas, las cuales están a la vez estructuradas por conectores como la negación, disyunción, implicación y bicondicional. Estos conectores permiten representar afirmaciones mediante predicados, donde cada uno puede tener uno o más parámetros para representar la información como pueden ser las premisas $\mathrm{A}(\mathrm{x})$ o también $\mathrm{B}\left(\mathrm{X}_{1}, \mathrm{X}_{2}, \ldots \mathrm{X}_{\mathrm{n}}\right)$ [17]. Como parte atómica de esta lógica se encuentran las clases, las cuales representan conceptos o entidades que se utilizan en la ontología. Estas son representadas mediante instancias o elementos individuales de una clase en particular. Los predicados que reúnen 2 o más elementos son conocidos como relaciones y finalmente se encuentran los axiomas formales, los cuales son modelamientos de afirmaciones que se consideran siempre verdaderas y que son utilizadas para validar ciertos predicados y a la vez realizar deducciones.

\subsection{Metodología}

Las herramientas usadas para la realización de este trabajo incluyen el lenguaje OWL y la versión de software Protege 5.0 con la cual se construyó la ontología junto con el razonador Pellet para verificar su consistencia. Para la realización de la ontología, se eligió la metodología propuesta por [7] debido a que es sencilla para la realización de este tipo de modelos.

Las fases generales que incluye esta metodología son las siguientes:

Fase 1: Determinar el dominio de la ontología: Consiste en elegir el tema base de la ontología, el cual en este trabajo corresponde a la pasantía.

Fase 2: Enumerar los conceptos importantes de la ontología: Se definen los términos principales del modelo. Para este caso se utilizaron conceptos como pasantía, trabajo de grado, estudiante, tema, entre otros.

Fase 3: Definir clases de la ontología y su jerarquía: Se organizan las entidades del modelo de manera jerárquica. En esta etapa se pueden usar elementos gráficos para obtener una representación de la ontología más entendible.

Fase 4: Definir las propiedades de las clases: Se enumeran las relaciones existentes entre las clases. Esto se realizó en el lenguaje OWL.

Fase 5: Detallar las propiedades de las clases: Se representan algunas características propias de las relaciones establecidas previamente.

\subsubsection{Planteamiento de la ontología}

La ontología propuesta presenta los conceptos y relaciones principales de la pasantía como una de las modalidades de grado en instituciones de educación superior. Para la obtención de este modelo se siguieron los pasos presentados a continuación, correspondientes a las etapas $a, b$ y $c$ de la metodología base utilizada para la creación de la ontología [7]:

\section{A. Enumeración de los conceptosimportantes de la ontología} 0122-820X ISSN 2422-5053 PP: 119-134

Para facilitar la comprensión de las inferencias a realizar, a continuación se presentan los conceptos relevantes de la representación 
No. 2

Julio - Diciembre 2015 ISSN 0122-820X

E-ISSN 2422-5053 PP: 119-134 ontológica de trabajos de grado en modalidad pasantía, los cuales están basados en documentos de instituciones de educación superior [18], [19]:

- Pasantía: Modalidad de trabajo de grado que permite a un estudiante aplicar en una empresa conocimientos de su formación académica.

- Trabajo de grado: Documento que elabora un estudiante para finalizar su proceso de formación de pregrado.

- Estudiante: Persona que realiza el trabajo de grado.

- Tema: Área de conocimiento al que pertenece un trabajo de grado.

- Jurado: Docente que califica la sustentación final de un trabajo de grado.

- Director: Docente que guía a un estudiante para realizar su trabajo de grado.

- Revisor: Docente que emite un concepto sobre la viabilidad de una propuesta o anteproyecto de trabajo de grado.

- Anteproyecto: Documento que contiene las características y metas principales del trabajo de grado a desarrollar por parte del estudiante.

- Director interno: Docente perteneciente a la universidad, encargado de guiar el desarrollo de trabajo de grado de un estudiante.
- Director externo: Profesional que labora en la empresa donde será desarrollado el proceso de pasantía y está encargado de guiar las actividades a realizar por el estudiante.

- Empresa: Entidad legalmente constituida donde es desarrollada una pasantía.

\section{B. Definición del modelo ontológico.}

El modelo ontológico puede ser representado mediante conceptos y sus correspondientes relaciones, los conceptos computacionalmente son equivalentes a estructuras de clases que representan jerarquías o relaciones de asociación, en la parte inferior la relación Subclase presentada en los numerales 6,8 y 9 , indica que el primer concepto tiene como subclase el segundo concepto, mientras que relaciones como las definidas en los cuatro primeros numerales representan asociaciones.

1 Trabajo de grado-Estudiante: hechoPor

2 Trabajo de grado-Tema: aborda

3 Trabajo de grado-Revisor: validadoPor

4 Trabajo de grado-Jurado: evaluadoPor

5 Trabajo de grado-Director: dirigidoPor

6 Trabajo de grado-Pasantía: Subclase

7 Pasantía-Empresa: realizada en

8 Director-Director interno: Subclase

9 Director-Director externo: Subclase

10 Trabajo de grado-Anteproyecto: formaliza

Una representación mediante grafos como la que se ilustra en la Figura 1 puede ser más entendible para los seres humanos, en este caso los nodos representan conceptos tales como Estudiante, Director y Jurado, mientras que los arcos representan relaciones tales como hechoPor, validadoPor y evaluadoPor, permitiendo visualizar la asociación entre conceptos. 


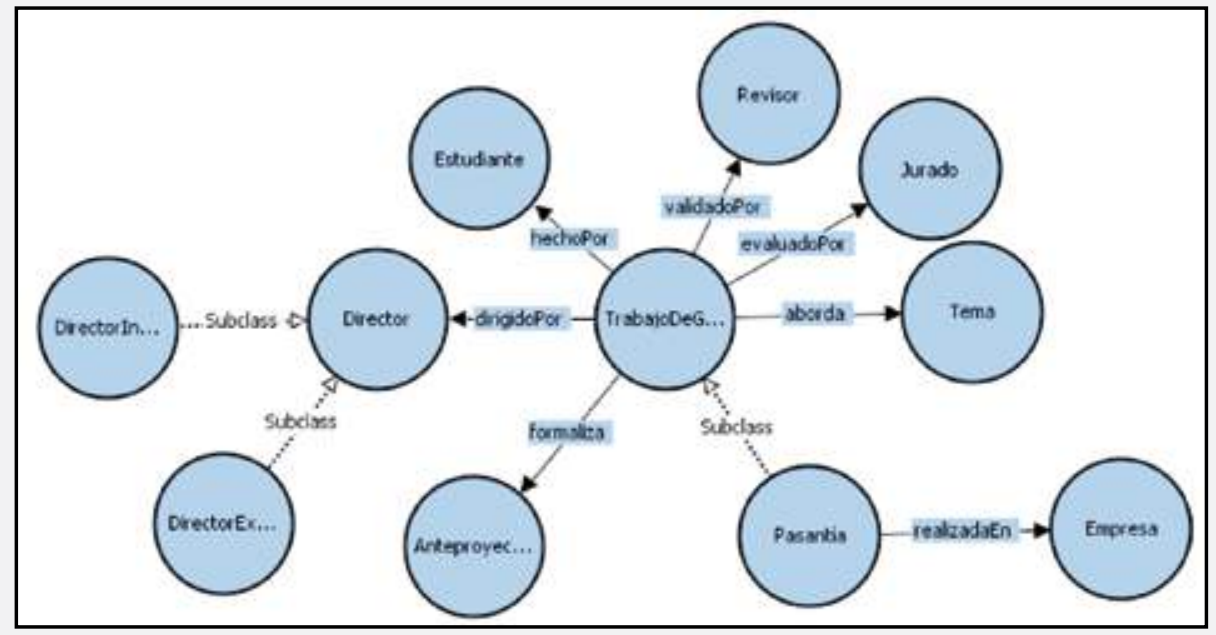

Julio - Diciembre 2015

ISSN 0122-820X

E-ISSN 2422-5053

La Tabla I explica las relaciones entre las entidades utilizadas, así como la cardinalidad de cada una de estas.

Tabla I. Dominio, rango y cardinalidad de las relaciones en la ontología.

\begin{tabular}{|c|c|c|c|}
\hline Relación & Dominio & Rango & Cardinalidad \\
\hline aborda & Trabajo de grado & Tema & Simple \\
\hline hechoPor & Trabajo de grado & Estudiante & Múltiple \\
\hline validadoPor & Trabajo de grado & Revisor & Múltiple \\
\hline evaluadoPor & Trabajo de grado & Jurado & Múltiple \\
\hline dirigidoPor & Trabajo de grado & Director & Múltiple \\
\hline realizadaEn & Pasantía & Empresa & Simple \\
\hline formaliza & Trabajo de grado & Anteproyecto & Simple \\
\hline
\end{tabular}

Fuente: Autores

\subsubsection{Representación de la Ontología en OWL}

La representación descriptiva de una ontología puede realizarse desde la perspectiva de clases y sus relaciones, como en el caso de trabajo de grado hecho por un estudiante, así como desde la especificación de restricciones como clases disjuntas (como en un docente el cual solo puede ser director o jurado de un mismo trabajo de grado) y restricciones de cardinalidad (como un trabajo de grado es realizado por uno o hasta $n$ estudiantes). Cuando se requiere especificar restricciones, es necesario aplicar OWL-FULL ya que esta versión además de restricciones, permite la representación de reglas y la obtención de inferencias dado su alto nivel de expresividad semántica [10].

Una representación exhaustiva de la temática de pasantías es demasiado extensa y queda fuera del alcance de este documento. Para ejemplificar el uso de la notación, a continuación se ilustra su aplicación a los conceptos más relevantes, como se evidencia en los siguientes apartados, correspondientes a las etapas $d$ y $e$ de la metodología base [7]:

\section{A. Clases:}

Se utilizaron clases como trabajo de grado, pasantía, estudiante, tema, revisor, jurado, empresa, director, director interno y director externo. La representación general es de la siguiente forma:
1. Class(: TrabajoDeGrado)
2. Class(: Tema)
3. Class(: DirectorInterno)

\section{B. Individuos:}

Estos son instancias de las clases y se representan así:

1. Individual( :TrabajoDeGrado $: 1$ )

2. Individual( :Tema :IngenieríaDeSoftware )

3. Individual( :DirectorInterno :Mary

Rodríguez) 
No. 2

Julio - Diciembre 2015 ISSN 0122-820X E-ISSN 2422-5053 PP: 119-134

\section{Propiedades.}

Estas son las relaciones entre las clases. Cada una se representa mediante la estructura ObjectProperty, donde se especifica su dominio (domain) y su rango (range). Se tomaron las siguientes relaciones como las más relevantes:

1. ObjectProperty(: tieneUn domain (: TrabajoDeGrado) range (: Tema))

2. ObjectProperty(: dirigidoPor domain (: TrabajoDeGrado) range (: Director))

3. ObjectProperty(: hechoPor domain (: TrabajoDeGrado) range (: Estudiante))

4. ObjectProperty(: validadoPor domain (: TrabajoDeGrado) range (: Revisor))

\section{Subclases:}

Se representan con la estructura subClass Of:

1. SubClassOf( :TrabajoDeGrado: Pasantía)

2. SubClassOf( :Director :DirectorInterno )

3. SubClassOf( :Director :DirectorExterno )

\section{E. Relación 'uno de'}

Son enumeradores considerados como tipos de datos antes de ser considerados clases. Algunos ejemplos que se tuvieron en cuenta son los siguientes:

1. Class( : Tema

126
OneOf( :Testing :InteligenciaArtificial : Control ))

2. Class( : Director OneOf( : MaryRodríguez :DiegoBuitrago ))

\section{F. Clases disjuntas}

Algunas instancias solo pueden pertenecer a una única clase entre varias por ser mutuamente excluyentes. Como ejemplo se puede decir que un director únicamente puede ser interno o externo, por lo tanto una opción excluye a las demás, como se muestra a continuación:
disjointWith (:DirectorInterno :DirectorExterno)

Donde cada director existente es de un solo tipo como puede ser interno o externo de manera excluyente.

\section{G. Cardinalidad}

OWL permite establecer las cardinalidades entre las relaciones, permitiendo dar el número mínimo (minCardinality), máximo (maxCardinality) o la cantidad exacta (cardinality) de las instancias utilizadas por cada relación. La estructura base es de la forma (cantidad, relación, entidad rango). Algunas de estas se representan de la siguiente manera:

1. cardinality ( 1 :tieneUn :TrabajoDeGrado )

2. maxcardinality( 3 :hechoPor: TrabajoDeGrado )

El uso de herramientas como esta permite tener una idea más clara de la ontología construida, así como brindar la facilidad de uso adecuado en caso de que se desee ampliar o modificar de acuerdo a las condiciones impuestas por cada institución universitaria a nivel administrativo, buscando siempre mejorar los procesos de manejo de la información, debido a que en el futuro existirá una cantidad incalculable de esta y se necesitará agilidad en su gestión [20].

\subsection{Planteamiento de la lógica de predicados}

Dado que existen diferentes modalidades de trabajos de grado, en la primera parte se presentan las características generales del trabajo de grado y la segunda y tercera se centran en los procesos de iniciación y finalización de la modalidad como pasantía, respectivamente.

\subsubsection{Reglas generales.}

Se consultaron algunas universidades para obtener información generalizada sobre los trabajos de grado. Las reglas construidas en lógica de predicados fueron las siguientes: 
1 Todo trabajo de grado es dirigido por un director [18].

$\forall \mathrm{x}(\operatorname{trabajoDeGrado}(\mathrm{x})) \rightarrow \exists \mathrm{y} / \quad(\operatorname{director}(\mathrm{y}) \wedge$ esDirectorDe $(\mathrm{y}, \mathrm{x}))$

2 Todo trabajo de grado tiene un tema específico [18].

$\forall \mathrm{x}($ trabajoDeGrado $(\mathrm{x})) \quad \rightarrow \exists ! \quad \mathrm{t} / \quad($ tema $(\mathrm{t})$ $\wedge$ esTemaDe $(\mathrm{t}, \mathrm{x}))$

3 Todo trabajo de grado debe tener al menos un estudiante.

$\forall \mathrm{x}(\operatorname{trabajoDeGrado}(\mathrm{x})) \rightarrow \exists \mathrm{y} / \quad$ (estudiante $(\mathrm{y}) \wedge$ esTrabajoDeGradoDe (x,y))

4 Un estudiante tiene un único trabajo de grado.

$\forall \mathrm{x}($ estudiante $(\mathrm{x})) \rightarrow \exists ! \mathrm{t} /($ trabajoDeGrado $(\mathrm{t}) \wedge$ esTrabajoDeGradoDe $(\mathrm{t}, \mathrm{x}))$

5 Un director no puede ser revisor del mismo trabajo de grado.

$\forall \mathrm{x}, \mathrm{y} \quad$ (director(x) $\wedge$ trabajoDeGrado(y) $\wedge$ directorDe $(\mathrm{x}, \mathrm{y}) \wedge \forall \mathrm{z}($ trabajoDeGrado $(\mathrm{z}) \wedge$ esJuradoDe(x,z) $\vee$ esRevisorDe $(\mathrm{x}, \mathrm{z}))) \Rightarrow \mathrm{y} \neq \mathrm{z}$

6 Todo trabajo de grado en modalidad pasantía debe tener director interno $\mathrm{y}$ externo.

$\forall \mathrm{x}($ pasantía(x)) $\rightarrow \exists \mathrm{y}, \mathrm{z} \quad /($ directorInterno(y) $\wedge$ directorExterno $(\mathrm{z}) \wedge$ esDirectorInternoDe $(\mathrm{y}, \mathrm{x}) \wedge$ esDirectorExternoDe $(\mathrm{z}, \mathrm{x}))$

7 Todo anteproyecto tiene al menos un revisor.

$\forall \mathrm{a}($ anteproyecto(a) $) \rightarrow(\exists \mathrm{r} / \quad$ revisor(r) $\wedge$ esRevisorDe(r,a))

8 Un revisor puede ser recomendado para ser jurado

$\forall \mathrm{r} /($ revisor(r) $) \rightarrow \exists \mathrm{r} /$ puedeSerJurado(r)

Las actividades institucionales para la realización de un trabajo de fin de carrera en modalidad pasantía implican: oficialización, desarrollo y cierre. En el caso de pasantía las acciones de estas actividades se organizaron en dos procesos: iniciación y finalización. Se utilizaron tres estados para determinar la situación de la pasantía, explicados en la Tabla II.
Tabla II. Estados de una pasantía según el proceso

\begin{tabular}{|c|c|c|}
\hline NOMBRE & DESCRIPCIÓN & POSIBLES ESTADOS \\
\hline Oficialización & $\begin{array}{c}\text { Aprobación iniciación de la } \\
\text { pasantía. }\end{array}$ & $\begin{array}{c}\text { Viable, modificable o } \\
\text { no viable. }\end{array}$ \\
\hline Desarrollo & $\begin{array}{c}\text { Una vez finalizada y radicado } \\
\text { el documento de pasantía, los } \\
\text { jurados emiten concepto. }\end{array}$ & $\begin{array}{c}\text { Modificable o } \\
\text { sustentable. }\end{array}$ \\
\hline Cierre & $\begin{array}{c}\text { Estado de la socialización de } \\
\text { la pasantía. }\end{array}$ & Sustentado. \\
\hline
\end{tabular}

Fuente: Autores

\subsubsection{Reglas de la iniciación de la pasantía.}

Estas reglas describen detalladamente el proceso de iniciación de esta modalidad de grado.

1 Una pasantía se debe realizar en mínimo una empresa [19].

$\forall \mathrm{x}($ pasantía $(\mathrm{x})) \quad \rightarrow \exists \mathrm{y} / \quad($ empresa $(\mathrm{y}) \wedge$ empresaDePasantía(y,x))

2 Una pasantía debe tener tema y alcance [19].

$\forall x($ pasantía $(x)) \rightarrow \exists y, z / \quad(\operatorname{esTemaDe}(y, \quad x) \wedge$ alcanceDe $(\mathrm{z}, \mathrm{x}))$

3 El estudiante debe redactar anteproyecto de la pasantía [19].

$\forall \forall \mathrm{x}, \mathrm{y}($ estudiante(x)^pasantía(y)) ^ esTrabajoDeGradoDe

$(\mathrm{y}, \mathrm{x})) \rightarrow \exists \mathrm{a} /($ anteproyectoDe $(\mathrm{a}, \mathrm{x}))$

4 Se debe radicar el anteproyecto en el consejo curricular [19].

$\forall \mathrm{x}($ pasantía(x)) $\rightarrow \exists \mathrm{y} / \quad$ (anteproyectoDe(y,x) $\wedge$ radicación('ConsejoCurricular', y, fecha))

5 Una vez radicado, se asignan 2 revisores [19].

$\forall \mathrm{x}, \mathrm{y}($ pasantía(x) $\wedge$ anteproyectode $(\mathrm{y}, \mathrm{x})) \rightarrow \exists \mathrm{w}, \mathrm{z} /$ $($ revisorDe $(\mathrm{w}, \mathrm{x}) \wedge(\operatorname{revisorDe}(\mathrm{z}, \mathrm{x}) \wedge \neg \operatorname{igual}(\mathrm{w}, \mathrm{z}))$

6 Una vez asignados revisores, tienen 15 días para notificar el resultado al consejo curricular [19]. $\forall \mathrm{x}$ (anteproyecto(x) ^radicación('ConsejoCurricul ar', $\mathrm{x}$, fecha) $) \rightarrow \exists$ fecha2/(notificación('ConsejoCu rricular', $x$, fecha2) $\wedge$ fecha $2 \leq$ fecha $1+15$ )

7 El resultado puede ser viable, modificable o no viable [19].

$\forall x($ anteproyecto(x) $\wedge$ notificación('ConsejoCurr icular', $x, f)) \rightarrow \exists x /($ oficialización( $x$, 'viable') $\underline{\vee}$ oficialización( $\mathrm{x}$, 'modificable') $\underline{\mathrm{V}}$ oficialización( $\mathrm{x}$, 'noViable')) 
No. 2

Julio - Diciembre 2015 ISSN 0122-820X E-ISSN 2422-5053 PP: 119-134
8 Si es viable, el consejo curricular notifica a la oficina de pasantías, dando la fecha de aprobación [19].

$\forall x(\operatorname{anteproyecto}(x) \wedge$ oficialización( $x$, 'viable' $))$

$\rightarrow \exists \mathrm{x} /$ (notificación('OficinaDePasantías', $\mathrm{x}, \mathrm{f}$ ))

9 Luego de aprobada, se dice que la pasantía ha sido iniciada oficialmente [19].

$\forall \mathrm{x}, \mathrm{y}($ anteproyectoDe $(\mathrm{x}, \mathrm{y}) \wedge$ notificación('Oficina DePasantías', $\mathrm{x}, \mathrm{f})) \rightarrow$ iniciada(y, f)

\section{Unificación de predicados:}

Algunos predicados fueron unificados, mediante la adición de más parámetros al predicado. Esto con el objetivo de simplificar $\mathrm{y}$ facilitar el entendimiento de las reglas. Por ejemplo en la regla: Se debe radicar el anteproyecto en el consejo curricular, inicialmente se planteaba su representación de la siguiente forma:

lugarRadicación( 'ConsejoCurricular',y)^ fechaRadicación(y,fecha)^ lugarRadicación('Consejo Curricular', fecha)

Donde la primera parte corresponde a la existencia de la radicación del anteproyecto $y$ en el consejo curricular. La segunda parte corresponde a la verificación que el anteproyecto $y$ tiene una fecha de radicación denotada como fecha. La tercera parte corresponde a la existencia de una radicación en el Consejo Curricular en la fecha encontrada anteriormente. Una manera más sencilla y entendible de representación se presenta en la siguiente sentencia:

radicación('ConsejoCurricular', y, fecha)

En este caso se verifica la existencia de una radicación en el Consejo Curricular, donde se radicó el documento $y$ correspondiente al anteproyecto y que se realizó en una fecha denotada como fecha, la cual puede ser o no pre-establecida al momento de buscar el hecho. Se realizó igualmente para el predicado de tipo notificación $(x, y, f)$, donde $x$ corresponde al lugar donde se realizó la notificación, $y$ corresponde al documento enviado o el trabajo correspondiente a la notificación y finalmente $f$ representa la fecha del trámite realizado.

\subsubsection{Reglas de la finalización de la pasantía.}

Estas reglas explican la finalización de la modalidad pasantía.

1 El director interno, debe hacer una visita a la empresa a los dos meses del inicio de la pasantía [19].

$\forall \mathrm{x}($ pasantía(x) $\wedge$ iniciada(x,f) $) \rightarrow \exists \mathrm{d}, \mathrm{f}_{2} /(\operatorname{director}(\mathrm{d})$ $\wedge$ esDirectorDe $(\mathrm{d}, \mathrm{x}) \wedge \operatorname{visita}\left(\mathrm{d}, \mathrm{x}, \mathrm{f}_{2}\right) \wedge \mathrm{f}_{2} \geq \mathrm{f}+60 \wedge$ $\left.\mathrm{f}_{2} \leq \mathrm{f}+80\right)$

Para esta regla y la siguiente se utilizó un rango de 20 días.

2 El director interno, debe hacer una visita a la empresa a los 4 meses del inicio de la pasantía [19].

$\forall \mathrm{x}($ pasantía(x) $\wedge$ iniciada $(\mathrm{x}, \mathrm{f})) \rightarrow \exists \mathrm{d}, \mathrm{f}_{2} /(\operatorname{director}(\mathrm{d})$ $\wedge$ esDirectorDe $(\mathrm{d}, \mathrm{x}) \wedge \operatorname{visita}\left(\mathrm{d}, \mathrm{x}, \mathrm{f}_{2}\right) \wedge \mathrm{f}_{2} \geq \mathrm{f}+120 \wedge$ $\left.\mathrm{f}_{2} \leq \mathrm{f}+140\right)$

3 Existirá un acta por cada visita, llenando el formato de la pasantía, firmada por ambos directores [19].

$\forall \mathrm{x}($ pasantía(x) $\wedge \operatorname{visita}(\mathrm{d}, \mathrm{x}, \mathrm{f})) \rightarrow \exists \mathrm{a} /(\operatorname{acta}(\mathrm{a}) \wedge$ $\operatorname{actaDe}(\mathrm{a}, \mathrm{x}) \wedge \operatorname{actaFecha}(\mathrm{a}, \mathrm{f}))$

4 Luego de firmar estas actas, se da como finalizada la pasantía [19].

$\forall x\left(\operatorname{pasantía}(x) \wedge \operatorname{actaDe}(a, x) \wedge \operatorname{actaFecha}\left(a, f_{1}\right) \wedge\right.$ $\left.\operatorname{actaFecha}\left(\mathrm{a}, \mathrm{f}_{2}\right) \wedge \neg \operatorname{igual}\left(\mathrm{f}_{1}, \mathrm{f}_{2}\right)\right) \rightarrow$ finalizada $\left(\mathrm{x}, \mathrm{f}_{2}\right)$

5 Se deben radicar las 2 actas de las visitas en la oficina de pasantías [19].

$\forall \mathrm{x}($ pasantía(x) $\wedge$ finalizada(x,f)) $\rightarrow \exists \mathrm{a}, \quad \mathrm{b} /$ (actaDe $(\mathrm{a}, \mathrm{x}) \wedge$ actaDe $(\mathrm{b}, \mathrm{x}) \wedge$ radicación('OficinaD ePasantías', a, $\left.\mathrm{f}_{2}\right) \wedge$ radicación('OficinaDePasantí as', $\left.b, f_{3}\right) \wedge f 2 \geq f \wedge f_{3} \geq f \wedge \neg$ igual $\left.(a, b)\right)$

6 La oficina de pasantías debe enviar la información al consejo curricular [19].

$\forall \mathrm{x}($ pasantía $(\mathrm{x}) \wedge$ finalizada $(\mathrm{x}, \mathrm{f})) \rightarrow \exists \mathrm{f}_{2} /$ (notificaci ón('ConsejoCurricular', $\left.x, f_{2}\right) \wedge f_{2} \geq f$ )

7 Luego se asignan 2 jurados calificadores [19].

$\forall \mathrm{x}($ pasantía $(\mathrm{x}) \wedge$ finalizada $(\mathrm{x}, \mathrm{f})) \rightarrow \exists \mathrm{w}, \mathrm{z} /$ $(\operatorname{juradoDe}(\mathrm{w}, \mathrm{x}) \wedge(\operatorname{juradoDe}(\mathrm{z}, \mathrm{x}) \wedge \neg \operatorname{igual}(\mathrm{w}, \mathrm{z})))$ 
8 El resultado puede ser modificable o sustentable [19].

$\forall \mathrm{x}($ pasantía $(\mathrm{x}) \wedge$ finalizada $(\mathrm{x}, \mathrm{f})) \quad \rightarrow \exists \mathrm{x} /$ (desarrollo( $\mathrm{x}$, 'modificable') $\underline{\mathrm{V}}$ desarrollo(x, 'sustentable'))

$9 \mathrm{Si}$ es sustentable, los jurados, directores internos y estudiantes deben acordar fecha de sustentación [19].

$\forall \mathrm{x}($ pasantía $(\mathrm{x}) \wedge$ finalizada $(\mathrm{x}, \mathrm{f}) \wedge$ desarrollo(x,'sustentable')) $\rightarrow \quad \exists f_{2} \quad /$ (sustentación $\left(x, f_{2}\right) \wedge f_{2} \geq f$ )

10 A la sustentación los jurados le asignan una nota de 0 a 5 [19].

$\forall \mathrm{x}($ pasantía(x)^finalizada $(\mathrm{x}, \mathrm{f}) \wedge \operatorname{cierre}(\mathrm{x}$, 'sustenta do' $\left.^{\prime}\right) \rightarrow \exists y /($ notaDePasantía(y, $\left.x) \wedge y \geq 0 \wedge y \leq 5\right)$

11 A un acta se le asigna la nota respectiva [19].

$\forall \mathrm{x}($ pasantía $(\mathrm{x}) \wedge \operatorname{cierre}(\mathrm{x}$, 'sustentado' $)) \rightarrow \exists \mathrm{a}, \mathrm{y} /$ $($ notaDePasantía $(y, x) \wedge \operatorname{acta}(a) \wedge$ tiene $\operatorname{Nota}(a, y))$

Como se puede apreciar, mediante la lógica de predicados es posible representar cada una de las expresiones obtenidas inicialmente, dado que esta lógica permite dividir las oraciones en sujetos, verbos e incluso adjetivos [21]. De estas reglas es posible utilizar algunas con el objetivo de realizar inferencias y obtener información que no está explícitamente representada mediante ellas. Durante la obtención de reglas siempre se debe buscar expresar claramente los detalles de los procesos que se deben realizar durante cada actividad como lo son los trabajos de grado. Esto es muy importante porque muchas veces se omiten algunas características u objetivos no tan relevantes, llegando algunas veces al punto de hacer algunas suposiciones sobre estos y afectar las expectativas y requisitos de los interesados.

\section{Resultados y análisis}

Los resultados obtenidos en este trabajo se basan en las inferencias que pueden ser realizadas a partir de las reglas que describen el dominio de trabajos de grado en modalidad pasantía, la deducción de nueva información se realiza con base en hechos verdaderos que son registrados mediante aserciones en el modelo. A continuación, se presentan ejemplos de inferencias en lenguaje natural y en lógica de predicados, obtenidas mediante la lógica de predicados, en las primeras tres inferencias se plantean las premisas involucradas y la conclusión obtenida, las siguientes presentan el problema a solucionar y su respectiva representación:

\subsection{Inferencia sobre la relación Estudiante- Director.}

Premisas y conclusión obtenida en lenguaje natural:

$\mathrm{P}_{1}$ : Un estudiante tiene un único trabajo de grado.

$\mathrm{P}_{2}$ : Todo trabajo de grado es dirigido por un director.

$\mathrm{C}$ : Todo estudiante es guiado por un director.

Representación en lógica de predicados:

$\mathrm{P}_{1}: \forall \mathrm{x}($ estudiante $(\mathrm{x})) \rightarrow \exists ! \mathrm{t}($ trabajoDeGrado(t)^esTr abajoDeGradoDe $(\mathrm{t}, \mathrm{x}))$

$\mathrm{P}_{2}: \forall \mathrm{t} /$ trabajoDeGrado(t) $\rightarrow \exists \mathrm{d} /(\operatorname{director}(\mathrm{d}) \wedge$ esDirigidoPor $(\mathrm{t}, \mathrm{d})$ )

C: $\forall x(\operatorname{estudiante}(\mathrm{x})) \rightarrow$ esDirigidoPor $(\mathrm{x}, \mathrm{d})$

Ejemplo de aplicación con base en las instancias: estudiante Andrés, quien presenta el trabajo de grado con identificador 1, dirigido por el docente Pedro:

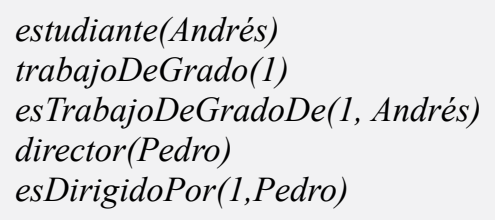

Con estas instancias es posible deducir que:

$$
\text { esDirigidoPor(Andrés, Pedro) }
$$

Esto permite inferir para todas las instancias que relacionan director y estudiante como en Pedro y Andrés, que el director no puede ser a la vez revisor, para lo cual no es necesario realizar nuevas especificaciones a nivel computacional, como sí tendría que realizarse en un modelo relacional.

\subsection{Inferencia sobre la relación Estudiante- Tema.} 2422-5053 : 119-134

Julio - Diciembre 2015 ISSN 0122-820X (20) 
No. 2

Julio - Diciembre 2015 ISSN 0122-820X E-ISSN 2422-5053 PP: 119-134 natural:

$\mathrm{P}_{1}$ : Un estudiante tiene un único trabajo de grado.

$\mathrm{P}_{2}$ : Todo trabajo de grado tiene un tema específico.

C: Todo estudiante debe tener un tema específico de interés.

En lógica de predicados:

$\mathrm{P}_{1}: \forall \mathrm{x}($ estudiante $(\mathrm{x})) \quad \rightarrow \exists ! \mathrm{t} /($ trabajoDeGrado $(\mathrm{t}) \wedge$ esTrabajoDeGradoDe $(\mathrm{t}, \mathrm{x}))$

$P_{2}: \forall t(\operatorname{trabajoDeGrado}(t)) \rightarrow \exists ! j / \operatorname{aborda}(t, j)$

C: $\forall \mathrm{x}(\operatorname{estudiante}(\mathrm{x})) \rightarrow \operatorname{aborda}(\mathrm{x}, \mathrm{j})$

Como ejemplo se tendrán en cuenta las siguientes instancias:

estudiante(Andrés)
trabajoDeGrado(1)
tema(pruebas)
esTrabajoDeGradoDe(1, Andrés)
aborda(1, pruebas)

Se infiere que:

aborda(Andrés, pruebas)

De la inferencia se obtiene que por cada estudiante debe existir por lo menos un tema que le corresponde, permitiendo encontrar inconsistencias al momento de registrar la información, sin realizar verificación mediante reglas adicionales.

\subsection{Inferencia sobre la relación Anteproyecto-Posible jurado.}

Premisas y conclusión obtenida en lenguaje natural:

$\mathrm{P}_{1}$ : Todo anteproyecto tiene al menos un revisor.

$\mathrm{P}_{2}$ : Un revisor puede ser recomendado para ser jurado.

C: Todo anteproyecto genera candidatos para ser jurado.

En lógica de predicados:

$\mathrm{P}_{1}: \forall \mathrm{a}($ anteproyecto(a) $\wedge$ aborda $(\mathrm{a}, \mathrm{t})) \rightarrow \exists \mathrm{r} /$ revisor $(\mathrm{r})$ $\wedge$ esRevisorDe(r,a)

$\mathrm{P}_{2}: \forall \mathrm{r} /(\operatorname{revisor}(\mathrm{r})) \rightarrow \exists \mathrm{r} /$ puedeSerJurado(r)

C: $\forall \mathrm{a}($ anteproyecto(a) $\wedge \quad \operatorname{aborda}(\mathrm{a}, \quad \mathrm{t})) \rightarrow \quad \exists \mathrm{r} /$ puedeSerJuradoDeTema(r, $t)$

Como ejemplo se tendrán en cuenta las siguientes instancias:

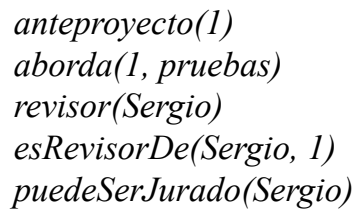

Con estas instancias es posible deducir que: puedeSerJuradoDeTema(Sergio, pruebas)

En este caso se infiere que el revisor Sergio puede ser un jurado apto para otros trabajos de grado sobre el tema de pruebas. Esto sucede para todos los revisores donde que cumplan con la instancia pruebas en la premisa aborda

\subsection{Inferencia aplicada a la búsqueda de documentos de un tema específico.}

Cuando un estudiante accede a repositorios de trabajos de grado de pasantía, ingresa una palabra relacionada con el tema para conocer los avances y trabajos ya realizados que puedan ser tenidos como referentes en su futuro trabajo de grado. Sin embargo, muchos de estos filtros de búsqueda generan resultados con base en el contenido ingresado en forma textual, obviando que el tema de interés puede ser descrito mediante otras cadenas de texto, con lo cual algunos resultados relevantes no serán presentados como resultado de la consulta. Esta situación se ilustra en la Tabla III:

Tabla III. Representación de temas y temas afines mediante variables

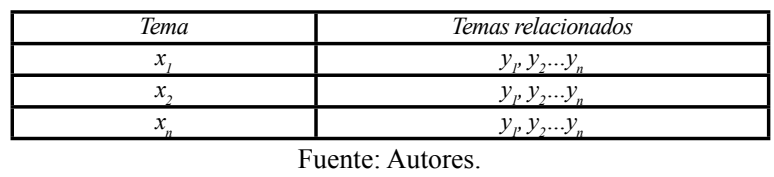

Donde cada término $\mathrm{x}_{\mathrm{i}}$ corresponde al nombre de cada uno de los temas generales de la carrera, mientras que cada término $y_{i}$ corresponde a los temas con los que están relacionados el tema $\mathrm{x}_{\mathrm{i}}$ en la columna de la izquierda.

Una vez construida la tabla anterior, por cada tema $\mathrm{x}_{\mathrm{i}}$, se genera una serie de hechos de la forma temaRelacionado $\left(x_{i}, y_{i}\right)$, obteniendo todas las relaciones entre cada uno de los 
temas de cada carrera, para luego obtener la inferencia correspondiente, como se presenta en la Tabla IV.

Tabla IV. Ejemplo real de temas y sus relaciones

\begin{tabular}{|c|c|}
\hline Tema & Temas relacionados \\
\hline 1. Pruebas de software & testing, validación, verificación. \\
\hline 2. Inteligencia artificial & robótica, control. \\
\hline & Fuente: Autores.
\end{tabular}

En este caso se relaciona el tema general de pruebas de software con los temas testing, validación y verificación, así como se relaciona el tema inteligencia artificial con los temas robótica y control.

Los hechos generados por la tabla son los siguientes:

temaRelacionado(pruebasDeSoftware, testing) temaRelacionado(pruebasDeSoftware, validación) temaRelacionado(pruebasDeSoftware, verificación) temaRelacionado(inteligenciaArtificial, robótica) temaRelacionado(inteligenciaArtificial, control)

Con lo cual, la inferencia obtenida para ser aplicada en la búsqueda de un documento es:

$\forall \mathrm{t}($ tema $(\mathrm{t}) \wedge$ tema $(\mathrm{j}) \wedge$ documento $(\mathrm{y}) \wedge \operatorname{esTemaDe}(\mathrm{j}, \mathrm{y})$ $\wedge$ temaRelacionado $(\mathrm{t}, \mathrm{j})) \rightarrow$ trabajoRelacionado $(\mathrm{y})$.

De esta forma al recibir como parámetro inicial un término específico, es posible obtener como resultado de búsqueda todos los trabajos que sean de un tema que haya sido considerado de relación con el tema inicialmente especificado, como se presenta en las instancias del siguiente ejemplo:

Como ejemplo se tendrán en cuenta las siguientes instancias:

tema(testing)
tema(pruebas)
documento(1)
esTemaDe(pruebas, 1)
temaRelacionado(testing, pruebas)

Con estas instancias es posible deducir que:

$$
\text { trabajoRelacionado(1) }
$$

Donde se infiere que el documento 1 acerca del tema pruebas es pertinente para ser mostrado como resultado de la búsqueda con la cadena de texto testing.

Julio - Diciembre 2015

Esta inferencia puede ser usada por un docente al momento de conocer el estado del arte sobre algún tema de investigación en el que desee profundizar así como por una universidad al momento de determinar si es adecuado llevar a cabo algún proyecto de tipo investigativo.

\subsection{Inferencia aplicada a la asignación de director a un trabajo de grado}

Otra situación en la que se pueden utilizar estas inferencias es al momento de asignar un director a cada trabajo de grado cuando el (los) estudiante(s) lo solicite(n). De esta manera se agilizará el proceso de asignar cada uno de los directores a estos trabajos, obteniendo resultados más rápidos y exactos en cuanto a la pertinencia del docente asignado en el tema del trabajo de grado. Esta inferencia se obtuvo de la siguiente manera: se estableció que todo trabajo de grado tiene un tema base, expresado mediante el hecho aborda(x, t) siendo $x$ un trabajo de grado y t un tema específico. De manera similar a la anterior inferencia, se buscó establecer una relación entre los temas de cada carrera, obteniendo la relación que si un docente domina un tema $\mathrm{x}$, es muy probable que posea conocimientos adecuados acerca de varios temas denotados como $\mathrm{y}_{1}, \mathrm{y}_{2} \ldots \mathrm{y}_{\mathrm{n}}$ por lo cual puede ser un docente adecuado para su asignación como director del trabajo de grado en cuestión. Esta relación se presenta en la Tabla $\mathrm{V}$.

Tabla V. Esquema de relaciones entre temas y temas afines

\begin{tabular}{|c|c|}
\hline Tema dominado & Temas dominados relacionados \\
\hline $\mathrm{x}_{1}$ & $\mathrm{y}_{1}, \mathrm{y}_{2} \ldots \mathrm{y}_{\mathrm{n}}$ \\
\hline $\mathrm{x}_{2}$ & $\mathrm{y}_{1}, \mathrm{y}_{2} \ldots \mathrm{y}_{\mathrm{n}}$ \\
\hline $\mathrm{x}_{\mathrm{n}}$ & $\mathrm{y}_{1}, \mathrm{y}_{2} \ldots \mathrm{y}_{\mathrm{n}}$ \\
\hline \multicolumn{2}{|c|}{ Fuente: Autores } \\
\hline
\end{tabular}

En este caso se utilizaron los mismos hechos de la inferencia anterior para obtener el resultado, donde un docente puede conocer otros temas si estos están relacionados directa o indirectamente con su área de interés principal $x$, mediante la premisa temaRelacionado $(x, y)$ siendo $y$ otro tema. Como ejemplo se puede 
No. 2

Julio - Diciembre 2015 ISSN 0122-820X E-ISSN 2422-5053 PP: 119-134 tomar un documento que requiere director sobre el tema de validación de software, representado por aborda (x, 'validación'). De esta manera, un docente que conozca el área de 'Pruebas de Software', puede ser candidato a dirigir el trabajo nombrado anteriormente. La inferencia obtenida para este caso es:

$\forall \mathrm{t}, \mathrm{j}, \mathrm{x}(\operatorname{tema}(\mathrm{t}) \wedge \operatorname{tema}(\mathrm{j}) \wedge \operatorname{documento}(\mathrm{x}) \wedge \operatorname{aborda}(\mathrm{x}, \mathrm{t}) \wedge$ temaRelacionado $(\mathrm{t}, \mathrm{j}) \wedge$ docente $(\mathrm{d}) \wedge$ dominaTema $(\mathrm{d}, \mathrm{j}))$ $\rightarrow$ puedeSerDirectorDe $(\mathrm{d}, \mathrm{x})$.

Al igual que en el caso anterior, se tiene en cuenta inicialmente un tema específico $\mathrm{t}$, correspondiente a un trabajo de grado, permitiendo obtener como resultado un docente que posiblemente domine el tema del trabajo de grado. Esta inferencia es aplicable una vez se haya verificado que no existen docentes con conocimientos en el tema específico del trabajo de grado, de lo contrario se asignaría directamente el docente encontrado con conocimientos en el tema específico buscado inicialmente.

Con esta inferencia es posible mejorar la probabilidad de acierto al momento de asignar un director con más correspondencia con el tema de cada trabajo de grado, donde muchas veces se hacen suposiciones en cuanto al conocimiento en determinadas áreas por parte de los docentes. Igualmente se reducirían tiempos de asignación debido a que se elimina la necesidad de conocer el historial de trabajos dirigidos por cada docente así como la consulta manual de dominios generales de cada docente. En este caso se hace necesario únicamente la existencia de un repositorio con las áreas específicas dominadas por cada docente, así como la especificación de las relaciones que pueden existir entre los temas que se le considera experto, como se presenta en el siguiente ejemplo aplicado a instancias:

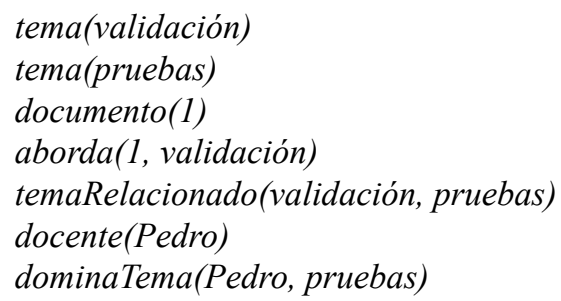

Con estas instancias es posible deducir que:

$$
\text { puedeSerDirectorDe(Pedro, 1) }
$$

Con esta inferencia es posible deducir que Pedro puede ser director del trabajo de grado del documento 1, asignando de esta manera para cada trabajo de grado un docente pertinente con su tema directo o uno relacionado de manera indirecta sin necesidad de ser definido explícitamente.

\section{Discusión:}

Algunas de las reglas utilizadas pueden ser representadas en diferente forma a nivel de lógica de predicados, conservando la equivalencia semántica. En el caso de la regla: un estudiante tiene un único trabajo de grado, se hizo la representación utilizando un cuantificador de tipo universal y uno de tipo existencial único de la siguiente forma:

$\forall \mathrm{x}($ estudiante $(\mathrm{x})) \rightarrow \exists ! \mathrm{t} /($ trabajoDeGrado $(\mathrm{t}) \wedge$ esTrabajoDeGradoDe $(\mathrm{t}, \mathrm{x}))$

De manera alterna, la regla anterior puede ser representada mediante el uso de un cuantificador universal junto con uno de tipo existencial así:

$\forall \mathrm{x}($ estudiante $(\mathrm{x})) \rightarrow \sim \exists \mathrm{t}, \mathrm{u} /($ trabajoDeGrado $(\mathrm{t}) \wedge$ trabajoDeGrado(u) $\wedge$ esTrabajoDeGradoDe(t,x) $\wedge$ esTrabajoDeGradoDe $(\mathrm{u}, \mathrm{x}) \wedge \neg$ igual $(\mathrm{t}, \mathrm{u}))$

Igualmente la regla del proceso de iniciación de la pasantía correspondiente a: se debe realizar en una única empresa, se representó mediante un cuantificador universal y uno de tipo existencial único de la siguiente forma:

$\forall \mathrm{x}($ pasantía $(\mathrm{x})) \rightarrow \exists ! \mathrm{y} / \quad($ empresa $(\mathrm{y}) \wedge$ empresaDePasantía $(\mathrm{y}, \mathrm{x}))$

Esta regla también puede ser representada como la regla anterior mediante un cuantificador universal y uno de tipo existencial de la siguiente forma:

$\forall \mathrm{x}($ pasantía $(\mathrm{x})) \rightarrow \sim \exists \mathrm{p}, \mathrm{q} /($ pasantía $(\mathrm{p}) \wedge$ pasantía $(\mathrm{q})$ $\wedge$ empresaDePasantía(p,x) $\wedge$ empresaDePasantía(q,x) $\wedge \neg \operatorname{igual}(\mathrm{p}, \mathrm{q}))$ 
Hacer un análisis detallado de las reglas expuestas, con el objetivo de encontrar la representación que sea óptima a nivel de procesamiento computacional en función de las operaciones a realizar como por ejemplo comparaciones y verificación de predicados, sería objeto de un futuro trabajo, esto con el objetivo de simplificar las expresiones, siendo el proceso de creación de formas lógicas un paso entre el análisis de tipo sintáctico de las expresiones y la obtención de estructuras semánticas de mayor profundidad y expresividad.

\section{Conclusiones}

La propuesta presentada para trabajos de grado modalidad pasantía es aplicable a otras modalidades de grado, como: monografía, proyecto de investigación o proyecto de emprendimiento, existiendo la posibilidad de seleccionar los conceptos como las temáticas para de esta manera obtener modelos adecuados en cada uno de los aspectos particulares que deben ser tenidos en cuenta. La lógica de predicados trabajada en repositorios permitiría realizar búsquedas con un mayor grado de pertinencia, mejorando la visibilidad de trabajos realizados anteriormente para ser ampliados o complementados en nuevas propuestas. El uso de modelos basados en la obtención de inferencias en problemas de manejo de altos volúmenes de información es muy factible, puesto que permite mejorar los resultados de actividades que facilitan en la institución, la toma de decisiones durante el desarrollo de procesos como los trabajos de grado por parte de los estudiantes. Mediante el uso de lógicas descriptivas es posible controlar las situaciones imprevistas de las instituciones como lo es la variación de la información a través del tiempo, al momento de agregar o eliminar directores, estudiantes o temas de cada carrera que aparezcan. Esta variación implica la actualización de los repositorios y la depuración dinámica de la información existente.

\section{Agradecimientos}

Se agradece de manera especial, los aportes generados por los integrantes del grupo de investigación INTECSE, así como a los integrantes del proyecto curricular de Ingeniería de Sistemas de la Universidad Distrital.

\section{Referencias}

[1] Teseo. Servicio de consultas. Marzo de 2015. [Online]. Disponible en: https://www.educacion.gob.es/teseo/ irGestionarConsulta.do.

[2] Cybertesis. Servicio de consultas. Marzo de 2015. [Online]. Disponible en: http:// cybertesis.unmsm.edu.pe/.

[3] SINAB. Repositorio institucional. Marzo de 2015. [Online]. Disponible en: http://www.sinab.unal.edu.co/.

[4] E. Abreo y C. Padilla, "Prototipo para recuperación semántica de información de proyectos de grado en ingeniería de sistemas con base en un repositorio ontológico", Redes de Ingeniería, vol. 3, no. 1, pp.109-118, 2012.

[5] J. Hendler, "Agents and the semantic web", IEEE Intelligent Systems, vol. 16, no. 2, pp. 30-37, march 2001.

[6] T. Gruber, "Toward Principles for the Design of Ontologies Used for Knowledge Sharing", International Journal of Human and Computer Studies, vol. 43, no. 5-6, pp. 907-928, Nov./Dec. 1995.

[7] N. Noy, y D. McGuinness, “Ontology Development 101: A Guide to Creating Your First Ontology". Marzo de 2015. [Online]. Disponible en: http//www. ksl.stanford.edu/people/dlm/papers/ ontology-tutorial-noy-mcguinnessabstract.html.
Julio - Diciembre 2015

ISSN 0122-820X

E-ISSN 2422-5053

PP: $119-134$ 
No. 2

Julio - Diciembre 2015

ISSN 0122-820X

E-ISSN 2422-5053 PP: 119-134

[9] OWL Web Ontology Language Guide. Marzo de 2015. [Online]. Disponible en: http://www.w3.org/TR/owl-guide/.

[10] S. Ramírez, Y. Alonso, V. Hernández, A. C. Arias y D. La Rosa, "Comparando UML y OWL en la representación del conocimiento: correspondencia sintáctica", Revista Española de Innovación, Calidad e Ingeniería del Software, vol. 6, no. 3, pp.87-90, 2010.

[11] S. Russell y P. Norvig, "Representación del conocimiento", en Inteligencia Artificial Un Enfoque Moderno, Ed. Aragón: España, 2004, pp. 401-402.

[12] I. Flores, "Introducción al razonamiento sobre ontologías", Centro de Ingeniería de Software y Sistemas, vol. 7, no. 1, pp. 17-19, 2011.

[13] D. Lorge, "Predicate logic for software engineering", IEEE Transactions On Software Engineering, vol. 19, no. 9, pp. 856-858, 1993.

[14] L. Floridi, "Open problems in the philosophy of information", Metaphilosophy LLC and Blackwell Publishing Ltd, vol. 35, no. 4, pp. 560561, july 2004.
[15] A. Ali y M. Khan, "Knowledge representation of urdu text using predicate logic", en International Conference on Emerging Technologies, Islamabad, 2010, pp.293-294.

[16] J. Mylopoulos y H. Levesque, "An overview of knowledge representation", ACM SIGMOD Record, vol. 11, no. 2, pp. 5-7, 1980.
[17] K. Onot, S. Kawanott, Y. F'ukazawat y T. Kadokurat, "A resolution method from predicate logic specification into executable code", en IEEE Conference Publications, Kauai, 1992, pp. 481-483.

[18] U. del Rosario. "Reglamento sobre procesos de grado". Abril de 2015. [Online]. Disponible en: http://www. urosario.edu.co/cpg-ri/Pasantias-Grado/ documentos/reglamento-de-procesosde-grado/.

[19] U.Distrital."Procedimientodeiniciación de pasantía como modalidad de trabajo de grado". Abril de 2015. [Online]. Disponibleen:http://www.udistrital.edu. co:8080/documents/47880/1b3595b88fld-4ebc-9256-8295e26d9579.

[20] U. Fayyad, G. Piatetsky y P. Smyth, "From data mining to knowledge discovery in databases". Marzo de 2015. [Online]. Disponible en: http://www. csd.uwo.ca/faculty/ling/cs435/fayyad. pdf.

[21] A. Ali y M. Khan, "Selecting Predicate Logic for Knowledge Representation by Comparative Study of Knowledge Representation Schemes", en International Conference on Emerging Technologies, Islamabad, 2009, pp.2324. 